\title{
RETRACTION
}

\section{Anatomy of the subthalamic nucleus, with correlation of deep brain stimulation}

TO THE EDITOR AND READERSHIP: On behalf of all authors, I respectfully request the retraction of our article, "Anatomy of the subthalamic nucleus, with correlation of deep brain stimulation" ( $\mathrm{J}$ Neurosurg, published online ahead of print on April 24, 2015; DOI: 10.3171/2014.10. JNS145). We rushed the preparation of the paper and not all authors were afforded the opportunity to verify the paper's accuracy. In reviewing the published article, we find that it contains numerous mistakes - too many to be corrected by an erratum notice.

There are errors in the text. Some examples include the following:

The subthalamic nucleus (STN) is described as superomedial to the red nucleus and the substantia nigra, whereas the STN is superior and anterolateral to the red nucleus and dorsal to the substantia nigra.

The ansa lenticularis is said to arise from the lateral portion of the globus pallidus internus (GPi), although in the next sentence the ansa lenticularis is described as arising from the medial portion of the GPi.

One sentence states that the thalamus is situated posterolateral to the substantia nigra, whereas the thalamus is located superior to the substantia nigra.

Another sentence states, "the fibers traveling between the red nucleus and STN are known as the habenular commissure at midline sections of the brain." In fact, the habenular commissure is located in front of the pineal gland and connects the habenular nuclei on both sides of the diencephalon.

A number of structures in the figures are mislabeled or not labeled at all. Examples include:

In Figure 1B the superior longitudinal fasciculus is labeled, but cannot be seen in the picture. In addition, the corona radiata is labeled as the internal capsule.

Figure 4C incorrectly shows the primary motor cortex as anterior to the premotor area.
In Figure 4D the structure labeled as the habenular commissure is the fasciculus retroflexus. The structure labeled as the fornix is the stria medullaris thalami.

Figure legends are not exempt from errors themselves. Some examples of this include the following:

Figure 2 is stated to be a medial view, but it is actually an inferior view. In addition, the legend states, "The thalamus is located posterolateral to the substantia nigra," which is incorrect. In fact, the thalamus is located superior to the substantia nigra.

Figure 4A: The legend states that the anterior commissure is posterior and the dentate rubrothalamic tract is anterior. This is incorrect.

Figure 4B: The legend states that the substantia nigra and red nucleus are clearly visible. However, the red nucleus is not labeled.

There is some mismatching of citations with statements in the Discussion. For example:

"The relationship between the volume of the STN and the total volume of the brain is proportionally similar in all humans. ${ }^{6}$ " Reference 6 does not correspond to this sentence.

Last, some general comments are in error. For example:

The sentence, "In clinical practice, DBS [deep brain stimulation] of the STN is a promising new surgical option for the treatment of advanced PD," is incorrect. The STN is a well-established target in the treatment of Parkinson disease.

We apologize to the editors and readers for the inaccuracies found in our paper. We appreciate the opportunity to retract this paper from the literature.

Akın Akakın, MD

Bahcesehir University School of Medicine, Istanbul, Turkey

\section{INCLUDE WHEN CITING}

Published online November 6, 2015; DOI: 10.3171/2015.10.JNS145r.

CAANS, 2016 